\title{
Two heads are better than one
}

This year's Nobel Prize in chemistry was a surprise to many scientists. The merit of the laureates was not disputed, nor was the importance of their discovery, the ubiquitin-mediated degradation of proteins. The surprise came from the decision to award the prize for this important biological insight not in medicine but in chemistry. This decision, subsequently praised by many, underscores the fundamental role played by chemistry in biological research. Twenty-five years after the discovery, as the chemistry community honors this landmark contribution to cell biology, the field of biology continues to need input from chemists. This apparently symbiotic relationship between the two communities, however, is often overshadowed by their troubling inability to communicate. Collaborative research across discipline boundaries presents a level of complexity that should not be underestimated, but biologists and chemists should recognize its enormous benefit and engage actively in interdisciplinary projects.

Although biology continues to focus on molecular aspects, biologists by and large still don't know how to make molecules and rely heavily on analytical technologies that have been developed by chemists. On their end, chemists seem more confident in their own ability to 'do biology.' Indeed, in the 'kit era' it is easier for chemists to carry out molecular biology experiments than it is for biologists to synthesize new compounds. However, the ability to cut and paste DNA or express a protein in bacteria does not necessarily obviate the need for a trained biologist's input to convert a neat chemical idea into a worthy approach that will benefit important biological problems.

A productive collaboration requires both parties to value its mutual benefits. On one hand, biologists need to make the effort to understand the power, and also the limitations, of chemistry for their own use. Too often, in interactions initiated by biologists, chemists are pegged as the 'reagent providers.' The 'let's hire a chemist to make a few molecules' attitude is bound to cause scientists to flounder on the road of collaboration.

On the other hand, an overly traditional conception among chemists has led to a lack of respect for contributions that members of their discipline make to the biological sciences. Chemical reactions that can be enormously useful to study biological processes are still often dismissed as 'boring' by those who do chemistry for the sake of chemistry. Another aspect of this con- servative attitude, most likely the by-product of a funding tradition, is the conventional preference among chemists for research projects involving small groups, in contrast to large-scale efforts in biology, where lab consortia have come into fashion. To be considered for tenure, a junior scientist in a chemistry department is most often expected to solely own a number of projects focused on 'exciting' chemistry. This expectation, of course, is likely to discourage young scientists who would otherwise be tempted to apply chemistry to the study of biological processes.

Funding bodies are becoming aware of the limitations that their traditional grant allocation system has imposed on the field of chemistry. For example, the National Science Foundation (NSF) has launched the Chemical Bonding Centers program in an effort to create collaborations that allow chemists to address 'big' problems. Although the focus of these new grants must be chemistry, this effort will hopefully contribute to a cultural change in favor of interdisciplinary collaborations. Additionally, in acknowledgment of the relevance of chemistry's contribution to other fields, the NSF has stressed additional criteria for grant allocation for several years, asking applicants to demonstrate the usefulness of their project beyond fundamental chemical insights.

Another recent trend is the creation of graduate programs in chemical biology within major academic institutions. These joint programs are designed to allow students interested in applying chemistry to the study of biology to learn in parallel what they would normally learn sequentially in the course of graduate and then postgraduate training. Hopefully, this new breed of graduates, having learned the language of both communities, will serve as mediators and lead research at the interface of the two disciplines.

With the aim of publishing "techniques for life scientists and chemists," this journal hopes to be the forum for such joint efforts, and its editors will suggest collaborative approaches when they deem it appropriate. Importantly, the methodological focus allows chemists and biologists truly equal recognition as they trade back and forth the leading and supporting roles. Trends in funding allocations and new graduate programs are encouraging in their recognition of collaborative research, and hopefully, academic tenure committees will in turn truly value participation in multidisciplinary projects. 